that specially applies to surgical procedures which are stijl sub judice. Mules' operation has been before the profession for nearly ten years, yet great differences of opinion still exist as to its value. The main object of the present communication is to elicit from members of the medical profession their views as to the advantages of evisceration of the eyeball and their experiences as to its disasters. I may preface $m y$ remarks by saying that the case to be narrated is the only one out of a series of thirty in which the operation has, in my practice, been followed by an untoward result.

A boy ten years of age became an inmate of the Ophthalmic School at Hanwell towards the latter end of 1891. Six years previously his left eye had been accidentally cut by a companion, and since then had been subject to periodical attacks of redness and of pain. The condition of the eye may be summed up in a few words. Perception of light doubtful. Tension -1 . The cornea was crossed by a cicatrix which engaged the iris and extended for three millimetres into the neighbouring sclera. No trace of a pupil was present, and the eye was tender and readily irritated by slight causes, such as exposure to light. On Jan. 7th, 1892, evisceration of the eyeball was performed under ether. A glass sphere (the smallest size but one) was introduced into the scleral cavity, and the wound was closed by three sutures of fine China twist, inserted in a vertical direction. The operation was done with the ordinary antiseptic precautions. A light dressing of iodoform and boric acid ointment was applied to the lids. During the course of the two following days reaction was marked by the usual signs-viz, slight rise of temperature, pain in the eye, swelling of the lids, and a certain amount of chemosis. The patient slept fairly and ate well. On Jan. 11th the chemosis had increased markedly and formed a yellowish fold, flecked with hæmorrhagic points. Some small white vesicles were observed upon the skin of the lids, the nature of which was doubtful. Iodoform was, however, discontinued. On Jan. 13th it was noted that the left preauricular gland had become swollen and tender. The chemosis was more extensive, and its appearance reminded one somewhat of a tomato protruding from the palpebral fissure. On this date numerous punctures with a narrow blade were made into the swelling, and were then followed by iced applications. On the next day - that is, a week after evisceration-it was found that the stitches had given way, thus exposing the glass sphere to view. The latter was therefore removed, together with the sutures, and the scleral cavity was douched out with a tepid sublimate solution ( 1 in 5000). Notwithstanding this measure, chemosis continued to increase, and by Jan. 20th a lake-coloured fold of conjunctiva lay on the cheek and measured $40 \mathrm{~mm}$. by $15 \mathrm{~mm}$. My experience of the operation having furnished me with nothing parallel to the foregoing symptoms, I wrote to Dr. Males, who advised that the eye should not be bandaged and that the patient should be allowed to go about armed with a pair of protective goggles; further, that lead lotion should be used three or four times in the course of the twenty-four hours. Dr. Mules' recommendations with regard to treatment were put into force on Jan. $22 \mathrm{nd}$-that is, fifteen days after the operation. By Feb. 6th the chemosis had become so far reduced as to permit the intermarginal space of the lower lid to be seen in almost its entire extent. Palpation showed that at this period a small hard mass, slightly tender to the touch lay in the depths of the orbit. On Feb. 24th the conjunctival protrusion measured $25 \mathrm{~mm}$. by $10 \mathrm{~mm}$. and could be seen to arise almost wholly from the lower lid. To shorten my story the chemosis, after undergoing some trifling diminution, reached a stationary condition, a small button of granulation tissue sprouted from the operation wound, and the nodule, already noted as occupying the orbit, remained without alteration. This state of things continued for ninety-three days after operation, when further steps were deemed necessary. Accordingly, ether was administered and the orbital stump was removed by dissection, during which operation a small quantity of fat was unavoidably taken away. It was observed that the muscles attached to the mass were hypertrophied, a remark that also applied to the optic nerve. Eight months after the second operation the following notes were made: "The left orbit is occupied by some seven folds of pale conjunctiva, which radiate from a central point, not unlike the spokes of a wheel. With a single exception the furrows between adjacent folds are not deep." The patient was last seen about five months ago-i.e., upwards of two years after the performance of evisceration. The conjunctiva was then a good deal thickened and was disposed in a bunch-like way. An artificial eye had been introduced on more than one occasion, but its use was ultimately abandoned. since it caused irritation of the stump, as shown by discomfort, redness, and discharge.

Mr. John Griffith, pathologist to the Royal Westminster Ophthalmic Hospital, was good enough to examine the stump removed at the second operation, and this was bis report.

"1. Macroscopio appearances.-To the naked eye and to the sense of touch the mass presented a very irregular nodular look. A button-like granulation sprouted from its anterior aspect, and the optic nerve entering it posteriorly was apparently enlarged. The soft tissues around the hard and nodular stump were constituted by the muscular, tendinous, and fascial structures of the neighbourhood. The irregular outline of the thickened sclera could be readily distinguished on section.

"2. Mioroscopic appearances. - The structural tissues of the stump are mainly fibro-cellular. The sclera has undergcne extensive hycerplastic changes, and is about five millimetres in thickness. The increase is mainly due to the organisation of inflammatory material, and many fibro-blastic cells are still present in the meshes of the fibrous matrix. The stone-like nature of the stump is accounted for by the fibrous condition of the hypertrophied sclera. The cavity of the latter contains fibro-cellular material, arranged in open meshwork and showing many large vascular channels. There is an almost complete absence of pigment cells, of which, however, a few may be recognised near the entrance of the optic nerve. The nerve, althongh quite distinct, is undergoing an extensive metamorphosis. Its axis cylinders are not to be distinguished, and its fibrous reticulum is denser and more cellular than normal. The granulation button is composed of loose fibrocellular tissue. The fascial and tendinous structures that surround the stump are thickened. Since no sections show muscular bundles the latter have probably been trimmed a way in preparing the specimen for the microscope.'

To explain the foregoing case one is bound, I think, to assume the existence of a septic infection, passing by the perforating veins of the sclera into the tissues of the orbit and there setting up inflammation. It is not impossible that septic changes were present prior to operation-that the altered nutrient and other conditions brought about by surgical interference roused into activity germs that had long lain dormant in the tissues. In other words, the organisws bad been introduced into the interior of the eyeball by the accident six years before. The alternative theory, that infection pccurred during operation, must of course be carefully considered. I would repeat, however, that the details of the operation and the antiseptic precantions were absolutely identical in this case with those in twenty-nine other cases that did well.

Welbeck-street, W.

\section{THE EFFECT OF CREASOTE ON THE VIRULENCE OF THE TUBERCLE BACILLUS. ${ }^{1}$}

BY W. KINGTON FYFFE, M.B. CANTAB., M.R.C.P. LOND., MEDICAL REGISTRAR TO ST. GEORGE'S HOSPITAL; LATE PATHOLOGIST AND MEDICAL REGISTRAR TO THE VICTORIA PARK HOSPITAL FOR DISEASES OF THE CHEST.

IT is, I think, a moot point amongst practical physicians as to whether the admitted good which comes to the phthisical when treated with creasote is due to any interference with the virulence of the organism, or simply to the fact that the drug, by its antiseptic qualities, stops fermentation taking place in the stomach, consequently improves digestion, and so aids in the assimilation of food. The whole question resolves itself, in fact, into a simple issue : Has creasote any effect on the virulence of the bacillus, or does it simply act by increasing the powers of resistance of the patient? Whilst admitting at once the power of creasote in the latter direction, I hope to be able to show that the drug, if given in sufficient dose, does certainly interfere with the virulence of the organism in a very marked degree.

There are three methods by which creasote is commonly administered at the Victoria-park Hospital : (1) as an inhalation,

1 A paper read before the Pathological Section of the British Medical Association at the Annual Meeting at Bristol this year. (From the laboratory of the Conjoint Board.) 
(2) by the mouth, and (3) by means of the creasote chamber. In the first method from ten to twenty drops of creasote are placed on a sponge inserted in the perforated zinc plate of an ordinary inhaler; this is tied like a respirator over the patient's nose and mouth, and left on for several hours in the day time ; often the instrument is worn all night. The second method is familiar to everyone. The third, so far as I know, was initiated by Dr. Arnold Chaplin, assistant physician to Victoria-park Hospital. A small room some eight or nine feet square is chosen; the patient, with his eyes protected by glasses and his nostrils plugged, is placed in it, and a few ounces of ordinary commercial creasote are then placed in an iron dish over a spirit lamp and heated. As the creasote becomes hot, fumes rapidly come off till the whole room is thick with vapour and the patient is breathing little else. He is kept in this atmosphere for an hour at a time. Such, then, are the three methods which I have attempted to investigate and compare.

It seemed possible that something might be learnt by frequent observation of the morphology of the bacillus and some comparison in numbers, size, or form instituted between the sputa of patients taking creasote and those taking other arugs. With this end in view the sputa of twenty cases of phthisis, some of whom were taking creasote and some the ordinary drugs, were examined twice a week for two months, and differences in the number of the bacilli and their morphological characteristics were noticed. This method was then relinquished because, as far as those observations went, they tended to show that the number of tubercle bacilli bore little or no relation to the condition of the patient's health, and that no distinction could be drawn, either from their number or their morphology, between the bacilli of those cases treated by creasote and those taking other drugs. The second plan adopted was that recommended by Dr. Delépine at last year's meeting of the British Medical Association-namely, that of injecting tuberculous sputum into the leg of a guineapig. If this be done it appears to be invariable that in a few days' time the popliteal glands are affected, then the inguinal, and finally the lumbar. At the end of fifteen days the spleen and liver are definitely diseased, and if kept for a month or five weeks the lungs succumb and the guinea-pig dies. There is, therefore, a definite normal course of events taking place, and, given that the resistance of the animals is the same, it is possible to gauge the virulence of the injection by the extent of the mischief done. Thus, if at the end of fifteen days, when normally the liver, spleen, and glands should be affected, instead only the glands are the seat of disease, it is fair to say that the virulence of the sputum is less than normal. The resistance of the guinea-pig may be neglected, since all guinea-pigs absorb the tuberculous polson with avidity, but that there should be no possibility of error only the smooth-haired variety of guinea-pig was used.

The first series of observations was made on patients who were not taking creasote internally, but were inhaling it. At the same time they were one and all taking cod-liver oil or hypophosphites. All of them had definite physical signs of phthisis, with bacilli in their sputum. The cases selected were those in an early stage, who showed evidence, by the stethoscope and their general condition, of fairly rapid change. Advanced cases were avoided, on the principle that no drug was likely to affect them. The sputum taken was that coughed up in the early morning. This was injected into guinea-pigs before treatment of any kind was begun, when the patients first came in. At the end of their two months' stay in the hospital another specimen of sputum was injected into other guinea-pigs, and the results were compared. In each case the animals were kept one month before being killed. The sputum of twelve such cases was used, and to save time I may state at once that in every case the glands, spleen, liver, and lungs were found to be affected in those guinea-pigs killed after the second injection, and that these lesions differed in no way from those produced by the first injection. Hence it may be taken that creasote given as an inhalation has no effect whatever upon the virulence of the bacillus. It is to be added that in eight of these cases the patients left the hospital, having gained, some two stone, some six, and one a stone in weight. Their general health was much improved, but their physical signs were unaltered. Such improvement cannot be pat down to the diminished virulence of the organism, but to the increased power of resistance in the patient.

The next series of cases was that of patients taking creasote by the mouth, and here the results were distinctly more en- couraging. The dose ordinarily given is from two to twelve minims, not more. With regard to the smaller doses, a guineapig was injected with the sputum of a tuberculons patient before treatment. In three weeks the animal died from tubercle. The patient was treated with two minims of creasote three times daily for two months, and at the end of that time a second inoculation was made. In this case the animal lived for seven weeks and then died from general tuberculosis, so that, whereas in the first case such was the virulence of the sputum that the guinea-pig died in eighteen days, after treatment by the internal administration of creasote in small doses it did not succumb for seven weeks. In a second specimen from another patient taking the same dose the animal injected with the untreated sputum was killed at the end of fifteen days and found to have all its organs affected with tuberculosis. Another one, inoculated with the sputam at the end of two months' treatment, and killed on the fifteenth day, showed tuberculosis of the popliteal, inguinal, and lumbar glands, but no affection of any of the internal organs. With larger doses of creasote given by the mouth the effect was still more striking. Thus, an inoculation was made on Feb. 9th with untreated sputum from a patient who was put on six minims of creasote three times a day; the guinea-pig died on the twentieth day, with the usual post-mortem appearances. A second inoculation was made in another one on the same day, and it was killed on the fifteenth day, with tubercle affecting the abdominal viscera and beginning to affect the lungs. At the end of two months' treatment two fresh inoculations were made, one in a guinea-pig which was kept and lived for nine weeks, and then died from tuberculosis, and one in a guinea-pig which was killed on the fifteenth day. Tubercle bacilli were found in the lumbar glands, but not in the viscera. The glands were inflamed, but not caseating. The bacilli were few in number and small in size, thin, and not congregating into colonies. An attempt to cultivate them in serum did not succeed. Inoculations were made from patients taking ap to twelve minims of creasote The longest time that any guined-pig lived was three months, which occurred on the inoculation of sputum from a patient who for the last three weeks of his sojourn in hospital had been taking ten minims of creasote three times daily -a longer period of life than in the case of inoculation of a spatum where the patient was taking twelve minims at the same rate. Furtber, the guinea-pig killed in the usual way on the fifteenth day, and inoculated from the sputum of the case taking ten minims, showed less lesion than the one inoculated with the sputum of the case taking twelve minims, in that the lumbar glands only were affected. while the spleen was definitely diseased in the other case. Still, one case can hardly be held to militate against the fact that in eleven cases it was abundantly proved that the greater the dose the greater was the diminution of the virulence of the poison.

It must not be supposed that the inoculations were successful in every case. In five cases, in all, abscesses formed at the seat of inoculation, with the result that in each case the guinea-pig succumbed far earlier-generally on the tenth day-than it would normally have done. Tubercle bacilli were found in the pus of the local abscess and in the popliteal glands, but not elsewhere in these cases. The condition of the spleen and blood showed that in these cases the guinea-pigs died from septicæmia.

The patients selected whose sputum was used, as in the case of the inhalations, were those who showed physical signs of early phthisis, which were rapidly spreading. In no case was the sputum from a very advanced form of the disease used. The sum of the results from inoculations of the sputum of patients taking creasote by the mouth goes to prove that the virulence of the organism is materially affected even when the dose is small ; it must be admitted. however, that, if the guinea-pig was only kept long enough, despite the dose of creasote the animal always died from tubercle in the end. It cannot, therefore, be maintained that, when the creasote is given even in twelve minim doses, the bacillus is actually killed: its power is only rendered less harmful.

With regard to patients who were treated by the creasote chamber, of these I am sorry to say that only three cases can be cited. They were all cases resembling in their physica signs those already referred to. An inoculation was made on Feb. 9th from the sputum of a man who had been undergoing treatment by the chamber for two weeks previously. The guinea-pig inoculated died tuberculous on April 24th-i.e., it lived eleven weeks. A second inoculation from this patient was made a month later, the patient being 
upon the same treatment, and the animal inoculated lived for fourteen weeks. Another inoculation was made on Mrarch 6th from a case already treated by this method for two months, and the animal died fifteen weeks later. A third inoculation was made from a fresh case, but unfortunately the guinea-pig died from septicæmia before any conclusion could be drawn.

These observations are incomplete in two points. I had not intended, at first, to make any inoculations from patients treated in the creasote chamber, and consequently I had to take the sputum at a time when the patients were in the middle or at the end of their treatment. I have, therefore, no experiment showing what the virulence of the sputum was before treatment was begun; and, secondly, the small number of the cases renders it impossible to draw a definite conclusion. I would, however, point out that in the first two inoculations from the same case there is a marked difference in the time the guinea-pigs lived-due, as I think, to the increase of the dose of creasote absorbedand that the two cases treated in the creasote chamber give a better result in the length of time the guinea-pigs livednamely, thirteen and fourteen weeks respectively - than did inoculations from patients taking creasote in any other way.

I should mention one or two supplementary experiments on the effect of the injection of creasote into animals already tuberculous. Two guinea-pigs were inoculated with tuberculous sputum in one leg; into the other leg ten minims of creasote were injected. This was followed by the injection of five minims twice a week. A third animal was injected with the tuberculous sputum alone as a control experiment. One guinea-pig succumbed at the end of a week, death being due to cellalitis from the irritation of the creasote. There was much inflammation at the seat of inoculation. The popliteal and inguinal glands were enlarged. Tubercle bacilli were found in the pus of the primary inoculation, but none in the glands. The second guinea-pig lived for sixteen days and then succumbed to cellulitis. Here, again, bacilli were found at the seat of inoculation, but not elsewhere, and there was absolutely no lesion of the spleen or liver, such as one would normally expect at the end of sixteen days. The control animal, killed on the sixteenth day, had extensive affection of the glands, spleen, and liver, and one or two spots in the lungs. These experiments were repeated twice afterwards, with the same results, only that the doses of creasote were smaller and the animals lived longer; in every instance, however, they succumbed to the irritation of the creasote setting up cellulitis. Again, a guinea-pig was injected with tuberculous sputum, and the disease allowed to take its course up to the fifteenth day. Creasote, at first in fire minim doses, was then injected every other day into the opposite leg. In all the cases (four in number) so treated creasote had no effect whatever, and the animals succumbed on or about the twentieth day; when, however, the creasote injections were begun at the end of the first week it was possible, in three cases, to keep the animal alive for a month, and in two cases for six weeks ; finally, the guinea-pigs died from cellulitis set up by the irritation of the creasote, post-mortem examination showing that the lesions had not extended beyond the limit of the lumbar glands.

Such experiments would seem to show conclasively that creasote has a definitely restraining effect on the poison of phthisis, provided the disease has not gone too far; and such a pathological experience is on a par with clinical evidence. With an advanced case of phthisis nothing curative can be done; taken in an early stage, it is possible, if not to cure, at any rate to prolong life.

To sum up. In the first series of cases - those that were taking creasote simply as an inhalation in addition to other drugs-no effect in the virulence of the disease was noted. In the second series, where creasote was administered by the mouth in doses varying from two to twelve minims three times a day, though when the smaller doses were given the diminution of the virulence was slight, yet when the larger amounts were reached there was an extremely marked diminution in virulence. In the third series it may be pointed out that the animals lived longer than in any of the other cases, though it is impossible to dogmatise from such a small number. Fourthly, creasote injected under the skin in tnberculous guinea-pigs, provided the disease was not too far advanced, had a markedly restraining effect. Repeated attempts were made to grow tubercle bacilli on serum from animals inoculated with tuberculous sputum from patients taking creasote in large doses either by the mouth or by means of the creasote chamber, but with no success.

In conclusion, I have to express my thanks to the senior staff at the Victoria Park Hospital for allowing me to use material from their cases for the purposes of this investigation, and in particular I am under an obligation to Dr. Arnold Chaplin for the use of sputum from his cases treated in the creasote chamber.

Duke-street, $\mathrm{W}$.

\section{NOTES ON A FATAL CASE OF POISONING} BY SEWER AIR.

BY H. W. WEBBER, M.D., B.S., L.R.C.P. LoND., M.R.C.S.ENG.

A FEMALE CHILD aged four years was seen on July 27th, 1894. She had been ill for two days, the present complaint being of sore-throat and difficulty in breathing. Immediately previous to this she, in common with the other children of the family, had suffered from what the parents described as " mumps," no medical man being called in, and while recovering from this illness the present trouble appeared. The parents complained of constant bad smells being noticed at the back premises, and on inspection the origin of these was found to be in a small courtyard at the back of the house, in which the cesspit of the adjoining house was situated. This was furnished with an "inspection hole," the cover of which, a flat stone, fitted badly, and from which sewer air escaped. It was said that when the cesspit became overfull the sewage forced its way beneath the cover into the courtyard. The courtyard was small and dark, and almost surrounded by the back premises of this and the adjoining house, which formed an angle with it. In this house also bad smells were complained of in the bathroom, and several nembers of a large family had for some time past suffered from anæmia and occasional sore-throats. The child looked very anæmic and ill. On examination very little could be seen of the mucous membrane of the throat, the tonsils, faucial interspace, and pharynx being obscured by a collection of muco-pus, rendered frothy by mixture with air. As far as could be made out, however, no membrane was anywhere present. The tongue was thickly coated with creamy-white fur; purulent discharge oozed from the anterior nares. The submaxillary glands were only slightly enlarged. The temperature was $102^{\circ} \mathrm{F}$. and the pulse 110 . A mixture containing four minims of tincture of perchloride of iron was given every four hours, and a boracic solution was applied to the throat and nose. Under this treatment the above-noted condition of the throat cleared up in three days, leaving the child weak and profoundly anæmic; syncope threatened if she stood on her feet or sat upright for any length of time. A severe attack happened on the evening of Aug. 1st. She was treated with a mixture of ammonia and ether every three hours. On Aug. 2nd the surface of the body was cold, the temperature was $98^{\circ}$, the pulse was 130 , feeble and of low tension, and the child was in the same low and anæmic condition. The tongue was clean, red, and moist at the tip, being brownish and rather dry on the dorsum. The heart sounds were feeble, the first sound differing littie from the second; the cardiac impulse was visible and diffuse, being best felt in the sixth space one inch internal to the nipple. The patient complained of pain in the abdomen on that day. On examination it was found to be moderately full; the liver was uniformly enlarged, smooth, slightly tender, the edge rounded, and reaching downwards to a level of two inches above the umbilicus; the dulness commenced above at the sixth rib, and tenderness was complained of on percussion over the lower ribs. The spleen was not palpable and its dulness was not increased. The temperature was $98^{\circ}$. On Aug. 4th the patient lay in much the same state, the surface of the body being cold, the pulse 140, and the temperature $98^{\circ}$. She was kept wrapped in a warmed blanket, and was fed at frequent intervals with milk, beef-tea, and brandy, the ether and ammonia mixture being continued. On Aug. 7th the child passed no urine for more than twelve hours; a specimen of this when examined was of a pale yellow colour acid, containing a moderate amount of lithates and about 8 per cent. of albumen (estimated by boiling). The edge of the liver now reached the level of the umbilicus; it was rounded and the surface of the viscus was smooth and swollen, distending the right hypochondriac and epigastric regions; it was tender. On Aug. 8th no urine was 